\title{
Per-pixel Rendering of Terrain Data
}

\author{
Taek Sang Jeong and JungHyun Han* \\ Department of Computer Science and Engineering, Korea University, Korea
}

\begin{abstract}
This paper presents a novel approach to terrain rendering, which mostly relies on GPU/shader rather than CPU. The most popular representation for terrain data is uniformly sampled height field. As the height field is stored as a texture map, it is directly accessible by a pixel shader. The pixel shader uses a ray casting algorithm, and the $\mathrm{CPU}$ and the vertex shader provide ray information to be passed to the pixel shader. Then, the pixel shader samples the ray, computes the intersection of the ray and the terrain surface, and finally determines the pixel color. The experimental results show the feasibility of the shaderintensive approach to real-time terrain rendering.
\end{abstract}

\section{Introduction}

Terrain rendering is essential for many applications such as 3D games, geographic information systems, virtual reality, flight simulation, etc. Applications usually require walk-through or fly-through navigation of the terrain in an interactive or real-time mode. The most popular representation for terrain data is uniformly sampled height field, often called digital elevation model[1]. The height field is a set of height or elevation data sampled in a uniform grid, and its simplicity has made it very popular in terrain visualization. Various approaches have been proposed in terrain rendering, and a recent survey can be found in [2]. In the wide spectrum of the proposed approaches, a common factor is that they all require a tremendous amount of CPU computation, for example, to do LOD(levels of detail) control, occlusion culling, etc.

The role of computer graphics hardware is performing the rendering pipeline, which is largely partitioned into vertex processing and fragment processing. The early graphics hardware handled only the fragment processing, but soon evolved to be able to handle vertex processing which includes transformation and lighting (T\&L). Recently, we have witnessed the graphical processing unit (GPU) revolution where the hardware capability has been exponentially growing and further the hardware has become programmable. The programmable pipeline is named shader. The shader program is a set of GPU instructions, and is partitioned into vertex shader (or vertex program) and pixel shader (or fragment program).

This paper proposes shader-based terrain rendering algorithms, which maximally utilize the powerful computing capabilities of the shader. Taking the

\footnotetext{
^ Corresponding author.
} 
rendering load off the $\mathrm{CPU}$, the $\mathrm{CPU}$ can do otherwise impossible missions, such as AI control in game applications.

\section{Why Shader for Terrain Rendering}

The original terrain data are often perceived too large to display at interactive frame rates. Therefore, various methods have been adopted that reduce the complexity of the terrain data but retain the image quality. The LOD(levels of detail) method has been popular as a suitable tool that selects only a subset of the height field points and produces a coarser mesh from the subset. For example, the near/important parts are represented by a large number of small polygons, and the far/unimportant parts by a small number of large polygons. Good examples of the LOD-based algorithms are found in [3][4][5].

The graphics hardware revolution makes people call the above-mentioned $C P U$-intensive approaches into question, i.e. the bottleneck in the terrain rendering process often lies in the CPU, which performs terrain simplification and LOD generation per each frame. In contrast, the increased computing capability of the GPU allows the GPU to be loaded with more work. Reflecting the changed computing environment, for example, Losasso and Hoppe[6] proposed so-called geo-clipmap approach, which adopts a fairly simple LOD scheme such that a finer resolution is generated for a region closer to the viewpoint, and a coarser resolution for a farther region. Then, the simple LOD terrain is passed to GPU, which handles the discontinuities between the different LOD levels of the terrain data.

Along the emerging trend, this paper proposes a shader-intensive terrain rendering approach, where the CPU does little or only a small amount of work, and almost all rendering jobs are assigned to the GPU, more precisely to the pixel shader. The proposed shader-intensive approach presents the terrain data represented as a texture map to the pixel shader. Then, the pixel shader renders the terrain data using ray casting. The traditional discrete ray casting algorithm is used, where a ray from the viewpoint to a screen pixel position is generated, and then the intersection between the ray and the terrain surface is computed.

\section{$3 \quad$ Rendering Process}

The overview of the rendering algorithm can be stated as follows. First of all, terrain envelope is computed off-line, which is defined as a coarse superset of the real terrain geometry. The bounding box of the terrain data is a kind of envelope, but is too loose. A tighter envelope example is shown in Fig. 1-(a).

In the vertex shader, the object-space coordinates of the envelope mesh vertices are output as texture values (using TEXCOORD0), and therefore the pixel shader obtains the interpolated object-space coordinates for each pixel position. Those coordinates are taken as the starting points for ray casting, as illustrated in Fig. 1-(b). Each ray is then sampled by uniformly-spaced points. Each sampled point is processed in order towards the terrain surface, as illustrated in 


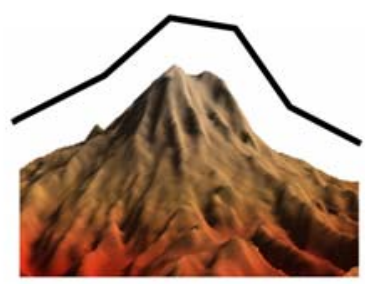

(a) terrain envelope

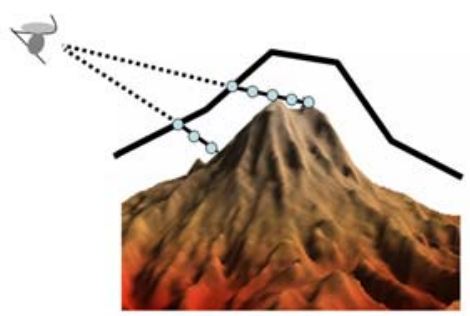

(c) ray sampling

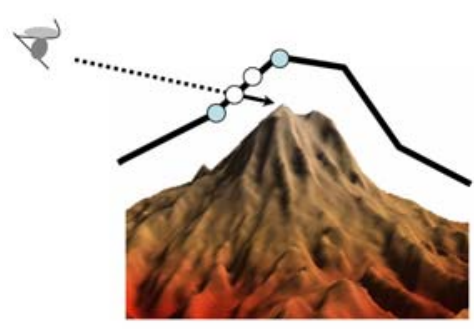

(b) starting points of ray casting

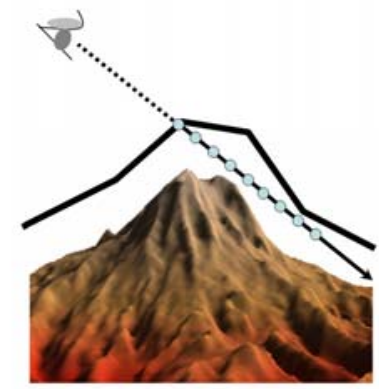

(d) no intersection with ray sampling

Fig. 1. Terrain envelope and ray casting

Fig. 1-(c). Each sampled point is tested if it lies below the terrain surface. If a point is found to be below the surface, the current and previous points are interpolated to determine the actual intersection position between the ray and the terrain surface.

For the intersection point, lighting is computed, i.e. Phong shading is implemented. For Phong shading, normals at the intersection points are obtained from the normal map, which is pre-computed from the terrain height map and passed to the pixel shader. The intersection position's (x,z)-coordinates are used to reference the texture map (real image texture!) of the terrain surface.

\section{Rendering Optimization}

As demonstrated in Fig. 1-(c), the required numbers of sampled points vary, depending on both the eye position and the ray direction. The major problem of the discrete ray casting is that it is hard to find the upper limit on the number of the sampled points. See Fig. 1-(d), where the ray is sampled for a finite number of iterations, but no intersection is found yet. Do we have to move further? Until when? There is no 'reasonable' upper limit. With a finite number of iterations, the intersection point between the ray and the target object is not guaranteed 
to be found even when it really exists. Only the infinite number of iterations guarantees it, but obviously we cannot do so.

Recently, shader-based ray casting has been explored in the domain of height field, and tackled the sampling problem. For example, Policarpo [7] proposed to use a combination of linear search and binary search, and Donnelly [8] proposed to use a so-called distance map in order to non-uniformly sample the ray. However, the intersection point is not guaranteed to be found in the proposed approaches. The rendering algorithm presented in this paper linearly searches the uniformly sampled points, but the intersection point is always found, if any.

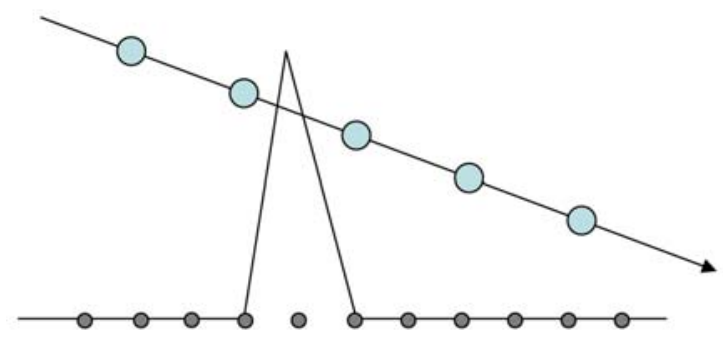

Fig. 2. Ray sampling resolution

Note that the height field data are organized in a uniform grid. In the proposed approach, the ray is uniformly sampled exactly at the resolution of the height field data. Then, it is guaranteed that we do not miss an intersection between the ray and the terrain surface. In contrast, it is not guaranteed as long as the ray samples are spaced farther than a single height texel, as shown in Fig. 2.

However, correctly setting the sampling resolution is not enough. We have to find a way to avoid infinite sampling. The solution is found by processing a bounded region at a time. For example, the entire height field can be partitioned into 9x9-sized blocks. Then, we need just 9 sampled points per a ray for processing the block. (Recall that the sampling resolution is equal to the height texel resolution.) If an intersection is found during the 9 iterations, color is computed for the intersection point. Otherwise, the ray segment can be safely discarded as the ray will be re-traced in the adjacent block(s). In other words, the undetermined pixel position will be processed again when an adjacent block is picked up.

The envelope is pre-computed, and the CPU's role is simply rendering the precomputed envelope. The tighter envelopes we have, the less pixels are processed in the pixel shader. For this purpose, we have adopted the simplification envelope approach $^{1}$ by Cohen et al. [9] Fig. 3-(a) and -(b) show a part of the height field data, and Fig. 3-(c) is its outer mesh, which is generated by adaptively offsetting the vertices (all along $+y$ direction) of the original mesh. The original mesh is taken as the inner mesh, and then mesh simplification is done only

${ }^{1}$ The original mesh's vertices are offset by a user-specified distance $\epsilon$ along its normal and opposite directions to produce outer and inner meshes, respectively. Then, the original mesh is simplified between the inner and outer meshes. Therefore, the simplified mesh is guaranteed to be within $\epsilon$ from the original mesh. 


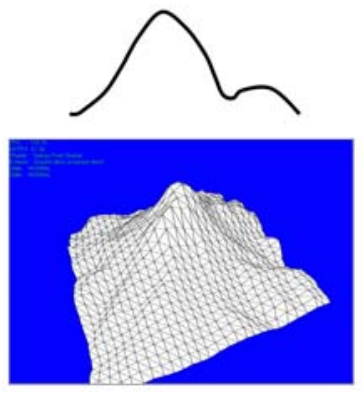

(a) original mesh

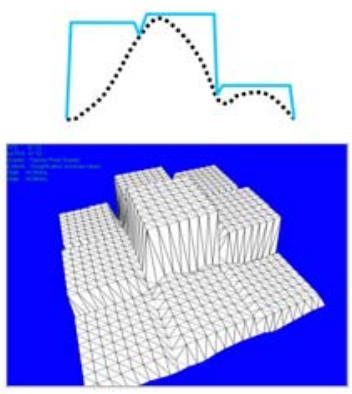

(b) outer mesh

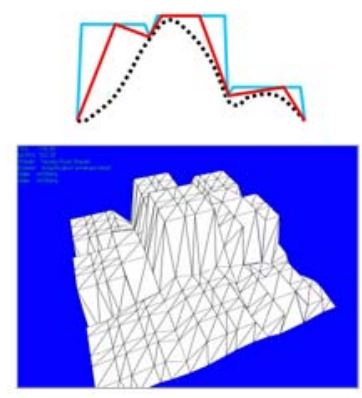

(c) simplified mesh

Fig. 3. Envelope construction

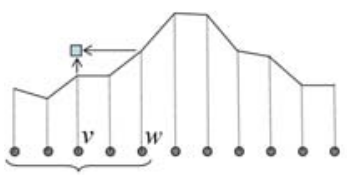

(a) height elevation

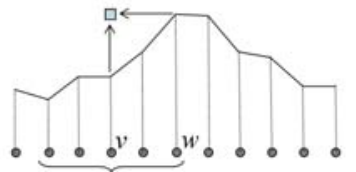

Fig. 4. Outer mesh construction

between the inner and outer meshes. For the sake of simplicity, the outer mesh itself is simplified in the current implementation, and the resulting simplified mesh, shown in Fig. 3-(d), lies between the original mesh (inner mesh) and the 'fine-resolution' outer mesh. Vertex decimation [10] is currently used as a simplification method.

Let us discuss the outer mesh construction process. See Fig. 4-(a) for an example in the cross section of the height field. Each vertex $v$ of the original height field has to be vertically (along $+y$ direction) offset to generate the outer mesh. To determine $v$ 's offset distance, consider a square (denoted by a brace in Fig. 4-(a)) centered at $v$, and take the height field vertex $w$ which is inside the square and has the highest elevation. The elevation of $v$ in the outer mesh is set to that of $w$. Such elevated or vertically offset vertices constitute the outer mesh, as shown in Fig. 4-(b).

Recall that we need bounded regions. For the current implementation, the size of a bounded region is $9 \times 9$. The outer mesh construction process is done per a bounded region. Furthermore, note that the boundary vertices of each region are not offset, as shown in Fig. 4-(b). By not offsetting the boundary vertices, the re-traced ray (discussed above) can be assigned the starting position for discrete ray casting.

\section{Experiment Results}

The terrain data set used for experiment is 1025x1025-sized Puget Sound[11]. Fig. 5 shows the height field data and its texture map. The height is represented by 1 byte, and therefore in the range of $[0,255]$. Fig. 5 -(a) is the gray-scale 
visualization of the height field data, where black(0) represents the lowest elevation and white(255) represents the highest elevation.

The experiment is implemented on Pentium $4(3.0 \mathrm{GHz})$ and NVidia GeForce 6800 Ultra with DirectX 9.0c and pixel shade ps_3_0. Unlike the previous profiles, ps_3_0 supports static and dynamic flow control such as iteration and branch, and therefore sampling of the ray becomes possible. Fig. 6 shows the snapshots of the rendered terrain from different viewpoints. The average frame rate is 78 fps (frames per second).

Fig. 7 compares two images: one is rendering with the polygon mesh obtained from the height field data, and the other is rendering with ray casting. Both of them have the comparable image quality.

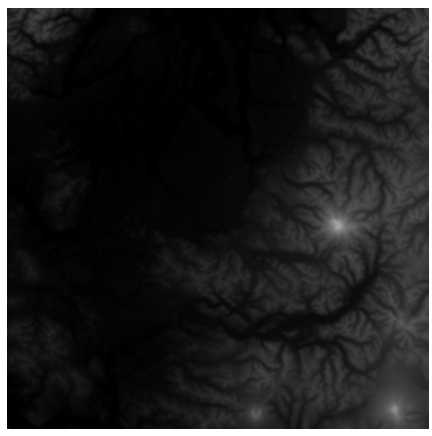

(a) height map

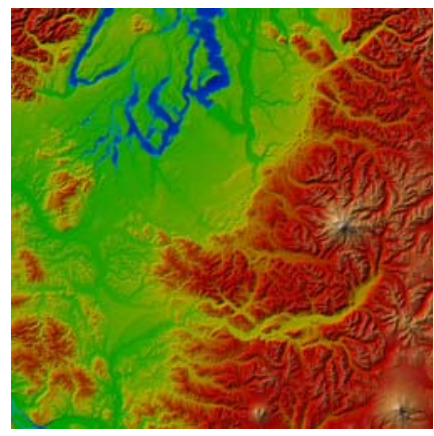

(b) texture

Fig. 5. Test data
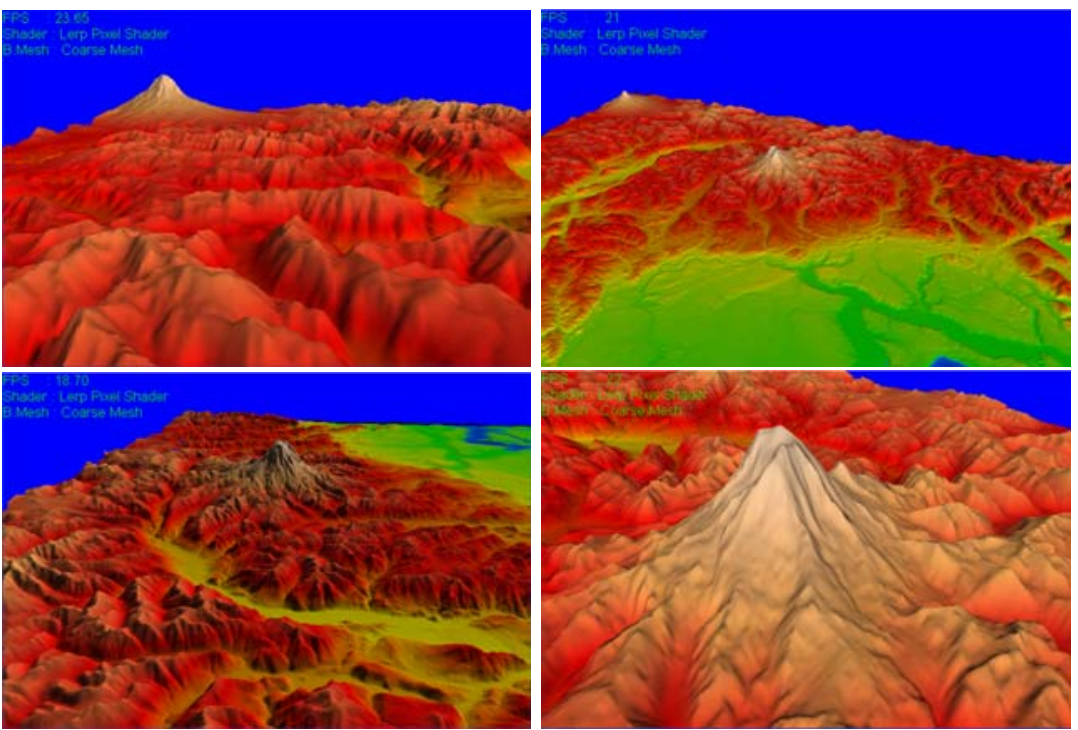

Fig. 6. Rendered images 


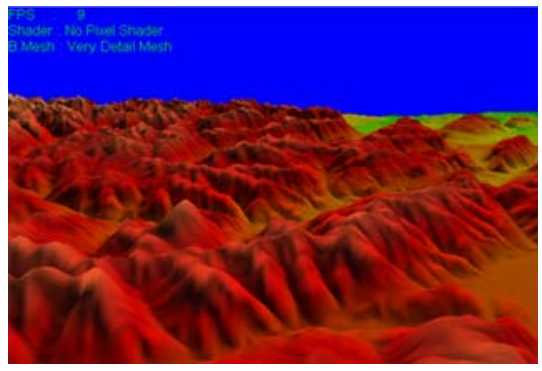

(a) mesh rendering

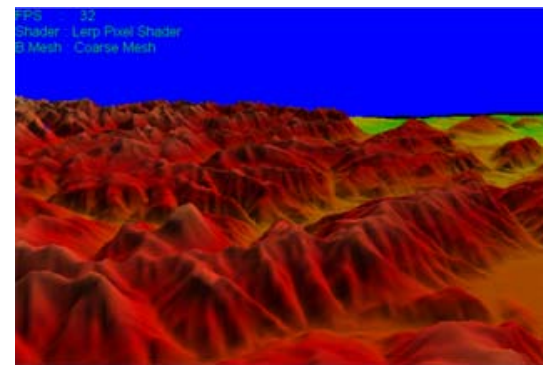

(b) ray casting

Fig. 7. Rendering result comparison

\section{Conclusion}

This paper presents a novel shader-intensive approach to terrain rendering. The height field stored as a texture map is directly accessible by a pixel shader. The pixel shader uses a ray casting algorithm, and the CPU and the vertex shader provide ray information to be passed to the pixel shader. The experimental results show the feasibility of the shader-intensive approach to real-time terrain rendering.

The earnest work of shader-based terrain rendering has been reported in [12] and [13]. The work by [12] proposes a simple application of shader. In the work by [13], however, the rendering process mostly relies on GPU/shader rather than CPU. Our approach follows such a shader-intensive approach as [13].

In the current implementation, the CPU does little work for the shader. However, the authors envision that the CPU could perform terrain data simplification and LOD generation unless they cause a bottleneck in the rendering process. Then, the simplified portion could be accommodated by a mipmap structure. Such a small CPU program can be plugged in the proposed framework ${ }^{2}$ where the main body of rendering is performed by the shader.

\section{Acknowledgements}

This research was supported by the Ministry of Information and Communication, Korea under the Information Technology Research Center support program supervised by the Institute of Information Technology Assessment, IITA-2005(C1090-0501-0019). This work was also sponsored and funded by Korea Game Development \& Promotion Institute as Korean government project (Ministry of Culture and Tourism).

${ }^{2}$ The traditional LOD construction algorithms cannot be moved to shader because the neighbouring vertex information which is essential for an LOD construction is not available to shaders. 


\section{References}

1. U.S. Geological Survey. http://www.usgs.gov/

2. R. Pajarola: Overview of Quadtree-based Terrain Triangulation and Visualization. UCI-ICS Technical Report No. 02-01, Department of Information \& Computer Science, University of California, Irvine, 2002.

3. P. Lindstrom, V. Pascucci: Terrain Simplification Simplified: A General Framework for View-Dependent Out-of-Core Visualization. IEEE Transactions on Visualization and Computer Graphics, Vol. 8, No. 3, July-September, pp. 239-254, 2002.

4. M. Duchaineau, M. Wolinsky, D. Sigeti, M. Miller, C. Aldrich, M. MineevWeinstein: ROAMing Terrain: Real-time Optimally Adapting Meshes. IEEE Visualization 1997, pp. 81-88, 1997.

5. S. Röttger, W. Heidrich, P. Slusallek, H.-P. Seidel: Real-time Generation of Continuous Levels of Detail for Height Fields. Central Europe Conf. on Computer Graphics and Vis., pp. 315-322, 1998.

6. F. Losasso, H. Hoppe: Geometry Clipmaps: Terrain Rendering using Nested Regular Grids. ACM SIGGRAPH, pp. 769-776, 2004.

7. F. Policarpo: Relief Mapping in a Pixel Shader using Binary Search. http://www.paralelo.com.br/arquivos/ReliefMapping.pdf.

8. W. Donnelly: Per-pixel Displacement Mapping with Distance Functions. GPU Gems 2, Addison-Wesley, pp. 123-136, 2005.

9. J. Cohen, A. Varshney, D. Manocha, G. Turk, H. Weber: Simplification Envelopes. ACM SIGGRAPH, pp. 119-128, 1996.

10. W. Schroeder, J. Zarge, W. Lorensen: Decimation of Triangle Meshes. ACM SIGGRAPH, pp. 65-70, 1992.

11. Puget Sound. http://www.cc.gatech.edu/projects/large_models/ps.html

12. Aaron Burton: Voxel Rendering with PS_3_0. ShaderX2 Shader Programming Tips \& Tricks with DirectX9, Wordware publishing, pp.161-171, 2004.

13. A. Asirvatham, H. Hoppe: Terrain Rendering using GPU-based Geometry Clipmaps. GPU Gems 2, Addison-Wesley, pp. 27-45, 2005. 